\title{
Effect of Organic, Inorganic and Integrated Nutrient Sources on the Yield and Its Attributes of Two Basmati Rice Varieties viz Type-3 and Taraori Grown in Tarai Regions of Uttarakhand India
}

\author{
Dipti Bisarya $^{1 *}$, D. K. Singh ${ }^{2}$, M. K. Nautiyal ${ }^{3}$, Deepti Shankhdhar ${ }^{1}$ and \\ S. C. Shankhdhar ${ }^{1}$
}

${ }^{1}$ Department of Plant Physiology, College of Basic Sciences \& Humanities, G. B. Pant University of Agriculture \& Technology, Pantnagar-263145 (Uttarakhand), India

${ }^{2}$ Department of Agronomy, College of Agriculture, G. B. Pant University of Agriculture \& Technology, Pantnagar-263145 (Uttarakhand), India

${ }^{3}$ Department of Genetics and plant Breeding, College of Agriculture, G. B. Pant University of Agriculture \& Technology, Pantnagar-263145 (Uttarakhand), India

*Corresponding author

\section{A B S T R A C T}

A field experiment was conducted at the organic block of breeder seed production center, G.B.P.U.A\&T, Pantnagar, Uttarakhand, India during the kharif season of 2015 and 2016 to

Keywords

Organic manure, basmati rice, FYM, VC, yield, SRI and physiology

Article Info

Accepted:

24 September 2018

Available Online:

10 October 2018 study the effect of different organic, inorganic and integrated nutrient sources on the growth, yield and yield attributes of two basmati rice varieties. The experiments were laid out in Split plot design replicated thrice with eight treatments as main plots viz., $\mathrm{T}_{1}$ : GM+FYM (AWD), $T_{2}$ : Organic (AWD), $T_{3}$ : FYM+VC (AWD), $T_{4}$ : SRI with FYM $(\mathrm{AWD}), \mathrm{T}_{5}$ : DSR + Soybean (LSI), $\mathrm{T}_{6}$ : Organic control $(\mathrm{CF}), \mathrm{T}_{7}$ : Chemical control (CF), $\mathrm{T}_{8}$ : Integrated $(\mathrm{CF})$ and two varieties Type-3 and Taraori in the sub plots. The plant height at maturity was highest in treatment $\mathrm{T}_{8}$ which was at par with $\mathrm{T}_{7}$ in both the years. The organic treatments $T_{1}$ and $T_{3}$ were at par with $T_{7}$ and $T_{8}$ in terms of productive tillers $/ \mathrm{m}^{-2}$, grain weight/panicle and thousand grain weight. However number of filled grains/panicle was non-significant in both the years. The grain yield ranged from 2.63-3.00 t/ha and the treatments $\mathrm{T}_{1}, \mathrm{~T}_{3}, \mathrm{~T}_{7}$ and $\mathrm{T}_{8}$ were at par in the in both the years 2015 and 2016. Further the results suggest that the organic manures such as vermicompost, FYM and green manures have equal potential in comparison to chemical and integrated fertilizers for rice production.

\section{Introduction}

Rice (Oryza sativa) is one of the most important cereal grains in the world today and serves as a staple food source for more than half of the world's population (Gross and
Zaho, 2014). About $90 \%$ of rice is grown and consumed in south and Southeast Asia (www.ricepedia.org IRRI, 2006). Although rice yields are still growing, the rate of growth has been declining; compound growth rate was $2.5 \%$ per annum (pa) during 1962-1979 
and declined to $1.4 \%$ pa during 1980-2011 (Adjao and Staatz, 2015). The cereal production forecast by Food and Agriculture Organization (FAO) in April-2018 indicated that out of the total cereal production, the contribution of rice (milled) is likely to be 503.9 million tonnes MMT. It is expected in future that the additional pressures will be build on the global food system because the demand for agricultural products is estimated to increase by about $50 \%$ by 2030 as the global population increases (Wheeler and Braun, 2013). Agricultural scientists are forced to produce more food within limited availability of cultivated land and water resources and particular socio-economic conditions. Over exploitation of vegetation and soil resources and adoption of inappropriate farming systems have resulted in land degradation and reduced crop production (Vaithiyanathan and Sundaramoorthy, 2016). The challenge of feeding a growing population expected to reach 9 to 10 billion people by 2050 while protecting the environment is daunting (Reganold and Wachter, 2016). A promising option is eco-functional intensification through organic farming, an approach where agricultural production aims at closing nutrient cycles, in which plant residues or manure from livestock are returned back to the fields, whereas neither synthetic fertilizers nor synthetic pesticides are applicable which minimizes negative environmental impacts (Lori et al., 2017). In the recent years organic agricultural land has increased almost five-fold as compared with 1999, when only 11 million hectares land area was under organic. In 2015, 6.5 million hectares, or almost 15 percent more were reported compared with 2014 (Willer and Lernoud, 2017). Although crop quality and yield depends on various factors, among which the nutrient sources and establishment methods play a vital role, there is very little information available on how rice yield is affected by different organic manures under different establishment methods and irrigation regimes. Therefore with this concept and the benefits of organic fertilizers in mind the present investigation was carried out to understand the effect of different organic and inorganic nutrient sources and different establishment methods and water management systems on morphological and agronomical parameters such as plant height, productive tillers $/ \mathrm{m}^{-2}$ grain weight/panicle, thousand grain weight, number of filled grains per panicle, grain yield, straw yield and harvest index of Taraori and Type- 3 basmati rice varieties.

\section{Materials and Methods}

\section{Experimental Site}

Field experiments were carried out at the Organic block of Breeder seed production Centre (BSPC) of G.B. Pant University of Agriculture and Technology, Pantnagar, Uttarakhand, India, during Kharif season 2015-2016. The experimental field was situated at $29^{\circ} \mathrm{N}$ latitude, $79.3^{\circ} \mathrm{E}$ longitude and $243.8 \mathrm{~m}$ above the mean sea level and lies in a narrow belt to the south of the foothills of Himalaya known as Tarai region.

\section{Weather data}

The minimum temperature, maximum temperature, sunshine hrs, rainfall and evaporation (Figure 1) were recorded for all meteorological weeks in 2015 \& 2016.

\section{Experimental details and Statistical Design}

The experiment was laid out in Split plot design with eight treatments as main plots and two basmati rice varieties viz Type-3 and Taraori as the sub plot with three replications of each treatment (Table 1).In GM treatment, Sesbania aculeata was incorporated at 50-55 days stage before the transplanting of rice along with $10 \mathrm{t} \mathrm{FYM/ha.} \mathrm{Manually} \mathrm{field} \mathrm{was}$ 
prepared with the help of spade followed by puddling and levelling. Two seedlings were translated in $20 \mathrm{~cm} \times 20 \mathrm{~cm}$ spacing. In the SRI treatment the twelve days old seedling were transplanted in a square grid pattern with one seedling per hill quickly and carefully so that roots get minimum trauma. In the DSR treatment direct seeded rice was intercropped with soybean and its yield was calculated as basmati rice grain equivalent yield. During both the years of the crop the weed management was done with the help of conoweeder after 15 days of transplanting (DAT) thereafter followed by two hand weedings at 30 and 45 DAT. Whereas in SRI treatment three weeding were done through conoweeder. Whereas in DSR, three hand weedings were done at 15, 30 and 45 DAT. Irrigations were managed as per the treatment requirements. The nursery preparation was done in the month of June in both the years and FYM $50 \mathrm{~kg} / 10 \mathrm{~m}^{2}$ was used as nutrient source. The nursery was sprayed with Leachate of vermicompost $(10 \%)+$ Neem cake $(10 \%)+$ Cow urine $(10 \%)$ along with Trichderma and Pseudomonas (@ each of 5 $\mathrm{gm} / \mathrm{l})$ after 15 days.

Selection of healthy seeds and control of disease was taken care from the initial stages for it the seeds were treated with salted water followed by the treatment of Pant bio agent-3 (Mixture of Trichoderma harzianum and Pseudomonas fluorescens) powder @ $10 \mathrm{~g} / \mathrm{kg}$ seeds and the seeds were kept moist under wet sack for germination. Insects and pests were controlled by spraying of $10 \%$ cow urine fortified with neem leaves at every 15 days interval. Whereas stem borers were controlled by the application of Trichocards (1 card for 1 acre area; 5 releases) and pheromone traps ( 5 mg pheromone per trap; 20 traps/ha; $20 \times 25$ $\mathrm{m}$ distance) within a week of transplanting. The lure was replaced after 30 days and the height of the trap was kept at $30 \mathrm{~cm}$ above the crop canopy.

\section{Statistical Analysis}

The statistical analysis of data for all the parameters was carried out with analysis of variance for split plot design. Critical difference (CD) was evaluated at $5 \%$ level of significance. The means were tested at $\mathrm{P}>$ 0.05 using STPR software designed at Department of Mathematics, Statistics and Computer Science, CBSH, G.B. Pant University of Agriculture \& Technology, Pantnagar, India.

\section{Results and Discussion}

\section{Morphological and Agronomical parameters}

\section{Plant height (cm)}

The plant height at maturity for two subsequent years i.e. 2015 and 2016 is summarized in the Table 2 . The plant height at maturity stage in 2015 was maximum (167 $\mathrm{cm})$ in $\mathrm{T}_{8}$ Integrated $(\mathrm{CF})$ and minimum (144 $\mathrm{cm})$ in $\mathrm{T}_{5} \mathrm{DSR}+$ Soybean (LSI). The plant height in the treatments followed the order $\mathrm{T}_{8}$ Integrated $(\mathrm{CF})>\mathrm{T}_{7}$ Chemical Control $(\mathrm{CF})>$ $\mathrm{T}_{2}$ Organic $(\mathrm{AWD})=\mathrm{T}_{4}$ SRI with FYM $\left(\right.$ AWD) $>\mathrm{T}_{6}$ Organic Control $(\mathrm{CF})>\mathrm{T}_{1} \mathrm{GM}+$ FYM $($ AWD $)=T_{3}$ FYM+VC (AWD) $>T_{5}$ $\mathrm{DSR}+$ Soybean (LSI). The maximum percent increase in plant height $(1.27 \%)$ was observed in $\mathrm{T}_{8}$ Integrated (CF) over $\mathrm{T}_{7}$ Chemical Control (CF) and maximum percent decrease $(-12.50 \%)$ in plant height was found in $\mathrm{T}_{5}$ $\mathrm{DSR}+$ Soybean (LSI) over $\mathrm{T}_{7}$ Chemical Control (CF). The treatments $\mathrm{T}_{8}$ and $\mathrm{T}_{7}$ were statistically at par with each other. However among both the basmati rice varieties significantly higher plant height at maturity stage was found in Type-3 $(160 \mathrm{~cm})$ than Taraori basmati rice variety $(152 \mathrm{~cm})$. In the year 2016 maximum plant height $(164 \mathrm{~cm})$ was observed in $\mathrm{T}_{8}$ Integrated $(\mathrm{CF})$ and minimum $(142 \mathrm{~cm})$ in $\mathrm{T}_{5} \mathrm{DSR}+$ Soybean 
(LSI). The plant height in the treatments followed the order $\mathrm{T}_{8}$ Integrated $(\mathrm{CF})>\mathrm{T}_{7}$ Chemical Control (CF) $>\mathrm{T}_{2}$ Organic (AWD) $>\mathrm{T}_{4}$ SRI with FYM $(\mathrm{AWD})=\mathrm{T}_{6}$ Organic Control $(\mathrm{CF})>\mathrm{T}_{3} \mathrm{FYM}+\mathrm{VC}(\mathrm{AWD})=\mathrm{T}_{1} \mathrm{GM}$ + FYM (AWD) $>T_{5}$ DSR + Soybean (LSI). The highest percent increase in plant height $(1.05 \%)$ was observed in $\mathrm{T}_{8}$ Integrated $(\mathrm{CF})$ over $\mathrm{T}_{7}$ Chemical Control (CF) and maximum percent decrease $(-12.36 \%)$ in plant height was found in $\mathrm{T}_{5} \mathrm{DSR}+$ Soybean (LSI) over $\mathrm{T}_{7}$ Chemical Control (CF). The treatments $\mathrm{T}_{8}$ and $\mathrm{T}_{7}$ were at par with each other. However among both the basmati rice varieties significantly higher plant height $(157 \mathrm{~cm})$ was found in Type-3 than Taraori $(150 \mathrm{~cm})$ basmati rice variety.

This could be due to the reason that the fundamental process of nutrient absorption by plants is well established. Irrespective of whether nutrients originate from organic or inorganic sources, plants are only capable of absorbing nutrients in certain forms. For example, nitrogen is only absorbed as nitrate $\left(\mathrm{NO}_{3}{ }^{-}\right)$ions or ammonium $\left(\mathrm{NH}_{4}^{+}\right)$ions and potassium only as $\mathrm{K}^{+}$ions. Water soluble inorganic fertilizers readily provide nutrients in these forms which could be easily taken up by the plants and utilized for their growth and development (Das and Mandal, 2015).

In a similar experiment higher plant height $(118 \mathrm{~cm})$ at the harvest stage was recorded with the application of $50 \% \mathrm{~N}$ through RDF + $50 \% \mathrm{~N}$ through vermicompost to rice variety GR 11 (Dekhane et al., 2014). In another study the application of organic and inorganic manures also increased the plant height significantly over control where maximum plant height was observed in treatment where RDF (150-90-60 NPK kg/ha) was applied. However different organic manures with $50 \%$ of RDF showed average plant height. Minimum plant height $(94.59 \mathrm{~cm})$ was recorded in control (Arif et al., 2015).
Plant height significantly increased due to different treatments compared to control treatment. At harvesting stage the highest plant height was recorded in treatment with farmyard manure @ 12.5 t/ha in combination with 100\% RDF (M4S1) followed by fish pond silt from desi poultry dropping @ 5 t/ha + vermicompost @ 5 t/ha combined with $100 \%$ RDF (M1S1). This increase in plant height in response to RDF might be primarily due to the improved vegetative growth and supplementary contribution of nitrogen (Kumar and Balusamy, 2017).

\section{Yield components}

\section{Productive tillers $/ \mathrm{m}^{-2}$}

Productive tillers $/ \mathrm{m}^{-2}$ at maturity (Table 3) of both the basmati rice varieties was significantly influenced by different organic, inorganic and integrated treatments in both the years. In the year 2015 maximum productive tillers $/ \mathrm{m}^{-2}$ (230) was observed in $\mathrm{T}_{5} \mathrm{DSR}+$ Soybean (LSI) and minimum (190) in $\mathrm{T}_{4} \mathrm{SRI}$ with FYM (AWD). The productive tillers $/ \mathrm{m}^{-2}$ in the treatments followed the order $\mathrm{T}_{5} \mathrm{DSR}+$ Soybean (LSI) $>\mathrm{T}_{8}$ Integrated $(\mathrm{CF})>\mathrm{T}_{1} \mathrm{GM}$ $+\mathrm{FYM}(\mathrm{AWD})=\mathrm{T}_{3} \mathrm{FYM}+\mathrm{VC}(\mathrm{AWD})>\mathrm{T}_{7}$ Chemical Control $(\mathrm{CF})>\mathrm{T}_{6}$ Organic Control $(\mathrm{CF})>\mathrm{T}_{2}$ Organic (AWD) $>\mathrm{T}_{4}$ SRI with FYM (AWD). The maximum percent increase in productive tillers $/ \mathrm{m}^{-2}(5.76 \%)$ was observed in $\mathrm{T}_{5} \mathrm{DSR}+$ Soybean (LSI) over $\mathrm{T}_{7}$ Chemical Control (CF) and maximum percent decrease $(-12.29 \%)$ in productive tillers $/ \mathrm{m}^{-2}$ was found in $\mathrm{T}_{4}$ SRI with FYM (AWD) over $\mathrm{T}_{7}$ Chemical Control (CF). However among both the basmati rice varieties higher productive tillers $/ \mathrm{m}^{-2}$ (218) was found in Type-3 than Taraori (212) basmati rice variety.

In the year 2016 maximum productive tillers $/ \mathrm{m}^{-2}$ (231) was observed in $\mathrm{T}_{8}$ Integrated $(\mathrm{CF})$ and $\mathrm{T}_{5} \mathrm{DSR}+$ Soybean (LSI) and 
minimum (192) in $\mathrm{T}_{4}$ SRI with FYM (AWD). The productive tillers $/ \mathrm{m}^{-2}$ in the treatments followed the order $\mathrm{T}_{5} \mathrm{DSR}+$ Soybean $(\mathrm{LSI})=$ $\mathrm{T}_{8}$ Integrated $(\mathrm{CF})>\mathrm{T}_{1} \mathrm{GM}+\mathrm{FYM}(\mathrm{AWD})>$ $\mathrm{T}_{7}$ Chemical Control (CF) $>\mathrm{T}_{3} \mathrm{FYM}+\mathrm{VC}$ (AWD) $>\mathrm{T}_{2}$ Organic (AWD) $>\mathrm{T}_{6}$ Organic Control $(\mathrm{CF})>\mathrm{T}_{4}$ SRI with FYM (AWD). However among both the basmati rice varieties higher productive tillers $/ \mathrm{m}^{-2}$ (222) was found in Type-3 than Taraori (217) basmati rice variety.

The use of organic manures and green manure would have resulted in improved soil quality it has also been previously reported that the fertile tillering also depends primarily upon soil physical conditions that were superior due to addition of poultry manure (Usman, 2003). Application of $50 \% \mathrm{~N}$ through RDF $+50 \% \mathrm{~N}$ through vermicompost recorded higher number of tillers per plant which were 8.7 and 12.1 at 45 DAT and at harvest time respectively, of rice variety GR 11 (Dekhane et al., 2014). Similarly the highest number of productive tillers $/ \mathrm{m}^{2}$ (409 and 422) was obtained in the treatment M4S1 (farm yard manure@12.5 t/ha in combination with $100 \%$ RDF) during both years respectively, which was followed by M1S1 (fish pond silt from desi poultry dropping @ 5 t/ha + vermicompost @ 5 t/ha with $100 \%$ RDF) (Kumar and Balusamy, 2017).

\section{Grain weight/panicle (g)}

Grain weight/panicle at harvest of both the basmati rice varieties under different organic, inorganic and integrated treatments in 2015 and 2016 are summarized in Table 3. In 2015 maximum grain weight/panicle was observed in $\mathrm{T}_{4}$ system of rice intensification (SRI) (1.61) and minimum (1.43 g) in $\mathrm{T}_{2}$ Organic (AWD). The grain weight/panicle in the treatments followed the order $\mathrm{T}_{4}$ SRI with FYM (AWD) $>T_{8}$ Integrated $(\mathrm{CF})>\mathrm{T}_{3}$ FYM+VC $($ AWD $)>T_{1}$ GM + FYM $($ AWD $)>$
$\mathrm{T}_{7}$ Chemical Control $(\mathrm{CF})>\mathrm{T}_{5} \mathrm{DSR}+$ Soybean (LSI) $>\mathrm{T}_{6}$ Organic Control $(\mathrm{CF})>\mathrm{T}_{2}$ Organic (AWD). The maximum percent increase in grain weight/panicle $(5.76 \%)$ was observed in $\mathrm{T}_{4}$ SRI with FYM (AWD) over $\mathrm{T}_{7}$ Chemical Control (CF) and maximum percent decrease $(-6.86 \%)$ in grain weight/panicle was found in $T_{2}$ Organic (AWD) over $T_{7}$ Chemical Control (CF). However among both the basmati rice varieties higher grain weight/panicle $(1.53 \mathrm{~g})$ was found in Taraori than Type-3 (1.51 g) basmati rice variety.

In 2016 maximum grain weight/panicle (1.67

g) was observed in $\mathrm{T}_{4}$ SRI with FYM (AWD) and minimum (1.42 $\mathrm{g}$ ) in $\mathrm{T}_{2}$ Organic (AWD).

The grain weight/panicle in the treatments followed the order $\mathrm{T}_{4}$ SRI with FYM (AWD) $>\mathrm{T}_{8}$ Integrated $(\mathrm{CF})>\mathrm{T}_{3} \mathrm{FYM}+\mathrm{VC}(\mathrm{AWD})>$ $\mathrm{T}_{1} \mathrm{GM}+\mathrm{FYM}(\mathrm{AWD})=\mathrm{T}_{7}$ Chemical Control $(\mathrm{CF})>\mathrm{T}_{5} \mathrm{DSR}+$ Soybean $(\mathrm{LSI})>\mathrm{T}_{6}$ Organic Control (CF) $>\mathrm{T}_{2}$ Organic (AWD). The maximum percent increase in grain weight/panicle $(8.37 \%)$ was observed in $\mathrm{T}_{4}$ SRI with FYM (AWD) over $\mathrm{T}_{7}$ Chemical Control (CF) and maximum percent decrease $(-7.67 \%)$ in grain weight/panicle was found in $\mathrm{T}_{2}$ Organic (AWD) over $\mathrm{T}_{7}$ Chemical Control (CF). However among both the basmati rice varieties higher grain weight/panicle $(1.55 \mathrm{~g})$ was found in Taraori than Type-3 (1.52 g) basmati rice variety.

This could be due to the reason that SRI has wider spacing between the plants due to which there is less below and above ground competitions for better grain filling, higher grain weight and more number of filled grains per panicle. Optimum supply of irrigation water with mechanical weeding resulted in higher nutrient availability subsequently resulting in better source to sink conversion and in turn enhanced the production of more total number of seeds and filled seeds per panicle (Lu et al., 2005). SRI method not only 
had the benefit of reducing the water requirement for rice cultivation but also increased the productivity. (Thiyagarajan et al., 2005). In similar study it was also recorded that significantly higher grain weight/panicle $(5.26 \mathrm{~g})$ was recorded in treatment with $45 \mathrm{~kg} \mathrm{~N} / \mathrm{ha}$ through $\mathrm{VC}$ among all other organic sources (Srivastava et al., 2016).

In another study maximum grain weight per panicle (1.44 g) was recorded in SRI treatment followed by chemical control (1.38 g) which was significantly higher with other treatments except GM + VC and DSR (Singh et al., 2017).

\section{Thousand grain weight (g)}

Thousand grain weight of both the basmati rice varieties under different organic, inorganic and integrated treatments in 2015 and 2016 are summarized in Table 4. In 2015 maximum thousand grain weight was observed in $\mathrm{T}_{3}$ FYM+VC (AWD) (21.37 g) and $\mathrm{T}_{8}$ Integrated (CF) $(21.37 \mathrm{~g})$ and minimum $(20.83 \mathrm{~g})$ in $\mathrm{T}_{4}$ SRI with FYM (AWD). The thousand grain weight in the treatments followed the order $\mathrm{T}_{8}$ Integrated $(\mathrm{CF})=\mathrm{T}_{3}$ FYM+VC (AWD) $>\mathrm{T}_{2}$ Organic (AWD) $>\mathrm{T}_{7}$ Chemical Control $(\mathrm{CF})>\mathrm{T}_{1} \mathrm{GM}$ + FYM $($ AWD $)>\mathrm{T}_{6}$ Organic Control $(\mathrm{CF})>$ $\mathrm{T}_{5}$ DSR + Soybean (LSI) $>\mathrm{T}_{4}$ SRI with FYM (AWD). The maximum percent increase in thousand grain weight $(1.81 \%)$ was observed in $\mathrm{T}_{3} \mathrm{FYM}+\mathrm{VC}$ (AWD) over $\mathrm{T}_{7}$ Chemical Control (CF) and maximum percent decrease $(-0.75 \%)$ in thousand grain weight was found in $\mathrm{T}_{4}$ SRI with FYM (AWD) over $\mathrm{T}_{7}$ Chemical Control (CF). However among both the basmati rice varieties higher thousand grain weight (21.72 g) was found in Taraori than Type-3 (20.41 g) basmati rice variety.

In 2016 maximum thousand grain weight $(21.10 \mathrm{~g})$ was observed in $\mathrm{T}_{1} \mathrm{GM}+\mathrm{FYM}$
(AWD) and minimum (20.87 g) in $\mathrm{T}_{5} \mathrm{DSR}+$ Soybean (LSI). The thousand grain weight in the treatments followed the order $\mathrm{T}_{1} \mathrm{GM}+$ FYM (AWD) $>\mathrm{T}_{4}$ SRI with FYM (AWD) $>$ $\mathrm{T}_{8}$ Integrated $(\mathrm{CF})>\mathrm{T}_{7}$ Chemical Control (CF) $>\mathrm{T}_{6}$ Organic Control $(\mathrm{CF})>\mathrm{T}_{3} \mathrm{FYM}+\mathrm{VC}$ $\left(\right.$ AWD) $>\mathrm{T}_{2}$ Organic (AWD) $>\mathrm{T}_{5}$ DSR + Soybean (LSI). The maximum percent increase in thousand grain weight $(0.19 \%)$ was observed in $\mathrm{T}_{4}$ SRI with FYM (AWD) over $\mathrm{T}_{7}$ Chemical Control (CF) and maximum percent decrease $(-0.91 \%)$ in thousand grain weight was found in $\mathrm{T}_{5} \mathrm{DSR}+$ Soybean (LSI) over $\mathrm{T}_{7}$ Chemical Control (CF). However among both the basmati rice varieties higher thousand grain weight (21.46 g) was found in Taraori than Type-3 (20.59 g) basmati rice variety.

It could be due to the reason that vermicompost is usually superior to ordinary aerobic compost in terms of nutritional status and microbial and enzymatic properties (Singh and Ganguly, 2005). Whereas green manure is a cheaper alternative to mounting price of fertilizer nitrogen and has become an effective technology in economizing the agriculture production system (Bana and Pant, 2000). In a similar experiment it was reported that there was a increase in 1000 grain weight from the plot receiving poultry manure and compost in combination with $50 \% \mathrm{RDF}$ and $100 \%$ recommended dose of fertilizer, which might be due to optimum accessibility of required plant nutrients as compared to other treatments (Arif et al., 2014).

Similarly thousand grain weight was recorded higher in SRI-organic + inorganic (21.8g, $18.7 \mathrm{~g}, 14.9 \mathrm{~g}$ and $14.8 \mathrm{~g}$ ) in Kharif season 2008 \& 2009 and Rabi season 2008 \& 2009 respectively (Gopalakrishnan et al., 2014). It was also reported that increase $(23.20 \%$ over control) in 1000 grain weight which was $21.12 \mathrm{~g}$ reported in Poultry manure+50\%RDF treatments (Arif et al., 2014). 
Fig.1 Weekly weather data of Pantnagar during the crop season (2015 \& 2016)

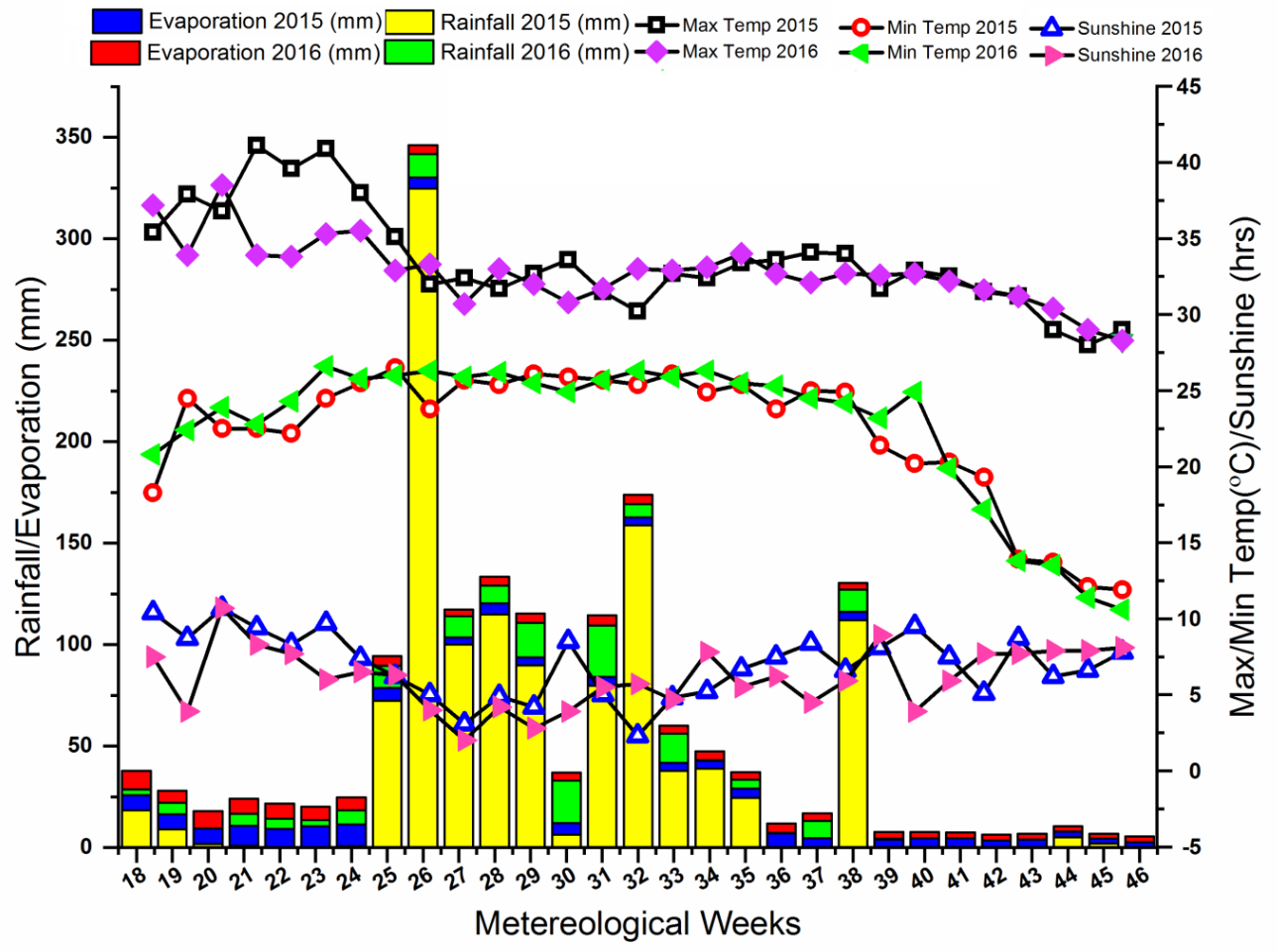

Fig.2 Effect of different treatments and varieties on rice grain yield (t/ha), straw yield (t/ha) and harvest index (\%) in 2015 \& 2016

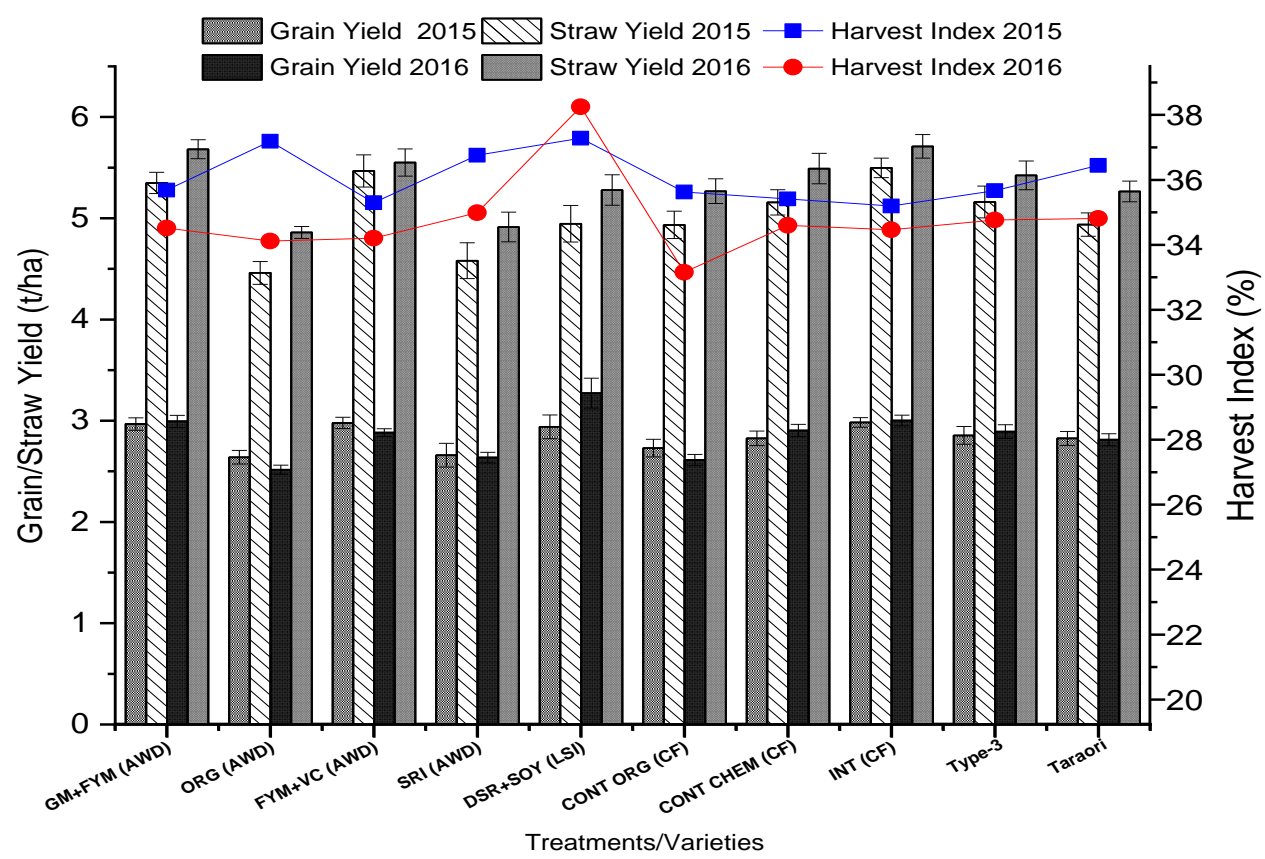


Table.1 Details of treatments

\begin{tabular}{|c|c|c|c|}
\hline $\begin{array}{l}\text { Treat. } \\
\text { No. }\end{array}$ & Abbreviation & $\begin{array}{l}\text { Water management } \\
\text { practice }\end{array}$ & Details \\
\hline $\mathrm{T}_{1}$ & $\begin{array}{l}\text { Green manuring + } \\
\text { FYM (AWD) }\end{array}$ & $\begin{array}{l}\text { Alternate wetting and } \\
\text { drying }\end{array}$ & $\begin{array}{l}\text { Transplaned rice using Sesbania aculeata as GM } \\
55 \text { days old crop@ @ } 16 \mathrm{tha}^{-1} \text { (fresh biomass) and } \\
\text { FYM@ } 5 \mathrm{tha}^{-1} \text { as nutrient source. }\end{array}$ \\
\hline $\mathbf{T}_{2}$ & Organic (AWD) & $\begin{array}{l}\text { Alternate wetting and } \\
\text { drying }\end{array}$ & $\begin{array}{c}\text { Transplanted rice using FYM @ } 10 \mathrm{t} \mathrm{ha}^{-1} \text { as } \\
\text { nutrient source. }\end{array}$ \\
\hline$\overline{T_{3}}$ & FYM+VC (AWD) & $\begin{array}{c}\text { Alternate wetting and } \\
\text { drying }\end{array}$ & $\begin{array}{c}\text { Transplanted rice using FYM @ } 10 \mathrm{t} \mathrm{ha}^{-1} \text { and VC } \\
@ 5 \mathrm{tha}^{-1} \text { as nutrient source. }\end{array}$ \\
\hline $\mathrm{T}_{4}$ & $\begin{array}{l}\text { SRI with FYM } \\
\text { (AWD) }\end{array}$ & $\begin{array}{l}\text { Alternate wetting and } \\
\text { drying }\end{array}$ & $\begin{array}{l}\text { System of Rice Intensification } \\
\text { (SRI) using FYM @ } 10 \mathrm{t} \mathrm{ha}^{-1} \text { as nutrient source. }\end{array}$ \\
\hline $\mathbf{T}_{5}$ & $\begin{array}{l}\text { DSR+Soybean } \\
\quad \text { (LSI) }\end{array}$ & Life saving irrigation & $\begin{array}{l}\text { Direct seeded rice crop + soybean using } \\
\text { FYM @ } 10 \mathrm{t} \mathrm{ha}^{-1} \text { as nutrient source. }\end{array}$ \\
\hline $\mathrm{T}_{6}$ & $\begin{array}{l}\text { Organic Control } \\
\text { (CF) }\end{array}$ & Continuous flooding & $\begin{array}{l}\text { Organic control using FYM @ } \\
10 \mathrm{t} \mathrm{ha}^{-1} \text { as nutrient source. }\end{array}$ \\
\hline$\overline{T_{7}}$ & $\begin{array}{l}\text { Chemical Control } \\
\text { (CF) }\end{array}$ & Continuous flooding & $\begin{array}{l}\text { Chemical control using } 70 \mathrm{~kg} \mathrm{~N} \mathrm{ha}^{-1}, 40 \mathrm{~kg} \mathrm{P}_{2} \mathrm{O}_{5} \\
\text { ha }^{-1} \text { and } 30 \mathrm{~kg} \mathrm{~K}_{2} \mathrm{O} \mathrm{ha} \text { as nutrient source. }\end{array}$ \\
\hline $\mathbf{T}_{8}$ & Integrated (CF) & Continuous flooding & $\begin{array}{l}\text { Integrated using } 35 \mathrm{~kg} \mathrm{~N} \mathrm{ha}^{-1}, 20 \mathrm{~kg} \mathrm{P}_{2} \mathrm{O}_{5} \mathrm{ha}^{-1} \\
\text { and } 15 \mathrm{~kg} \mathrm{~K} \mathrm{O} \mathrm{ha}^{-1} \text { along with farm yard manure } \\
\text { @ 5t/ha as nutrient source. }\end{array}$ \\
\hline
\end{tabular}

Table.2 Effect of different organic, inorganic and integrated nutrient sources on plant height of two basmati rice varieties at maturity stage in 2015 and 2016

\begin{tabular}{|c|c|c|c|c|}
\hline Treatments & $\begin{array}{c}\text { Plant height (cm) } \\
2015\end{array}$ & PCOC & $\begin{array}{c}\text { Plant height }(\mathrm{cm}) \\
2016\end{array}$ & PCOC \\
\hline$T_{1}: G M+F Y M(A W D)$ & 153 & -7.01 & 151 & -6.98 \\
\hline $\mathrm{T}_{2}:$ Organic (A WD) & 155 & -5.93 & 153 & -5.44 \\
\hline$T_{3}: F Y M+V C(A W D)$ & 153 & -7.11 & 151 & -6.52 \\
\hline $\begin{array}{l}\text { T4: SRI with FYM } \\
\text { (A WD) }\end{array}$ & 155 & -6.10 & 152 & -6.25 \\
\hline $\begin{array}{l}\text { T5: DSR + Soybean } \\
\text { (LSI) }\end{array}$ & 144 & -12.50 & 142 & -12.36 \\
\hline $\begin{array}{l}\mathrm{T}_{6} \text { : Organic Control } \\
\text { (CF) }\end{array}$ & 154 & -6.80 & 152 & -6.44 \\
\hline $\begin{array}{l}\mathrm{T}_{7} \text { : Chemical Control } \\
(\mathrm{CF})\end{array}$ & 165 & - & 162 & - \\
\hline $\mathrm{T}_{8}:$ Integrated $(\mathrm{CF})$ & 167 & 1.27 & 164 & 1.05 \\
\hline $\mathrm{CD}(\mathrm{p}=0.05)$ & \multicolumn{2}{|l|}{3.77} & \multicolumn{2}{|l|}{3.25} \\
\hline $\mathrm{SEm} \pm$ & \multicolumn{2}{|l|}{1.24} & \multicolumn{2}{|l|}{1.07} \\
\hline \multicolumn{5}{|l|}{ Variety } \\
\hline Type-3 Basmati & \multicolumn{2}{|l|}{160} & \multicolumn{2}{|l|}{157} \\
\hline Taraori Basmati & \multicolumn{2}{|l|}{152} & \multicolumn{2}{|l|}{150} \\
\hline $\mathrm{CD}(\mathrm{p}=0.05)$ & \multicolumn{2}{|l|}{1.51} & \multicolumn{2}{|l|}{0.93} \\
\hline $\mathrm{SEm} \pm$ & \multicolumn{2}{|l|}{0.50} & \multicolumn{2}{|l|}{0.31} \\
\hline
\end{tabular}

PCOC: Percent change over chemical control 
Table.3 Effect of different organic, inorganic and integrated nutrient sources on productive tillers $/ \mathrm{m}^{2}$ at maturity stage and grain wt/panicle $(\mathrm{g})$ of two basmati rice varieties in 2015 \& 2016

\begin{tabular}{|c|c|c|c|c|c|c|c|c|}
\hline Treatments & $\begin{array}{c}\text { Productive } \\
\text { tillers } / \mathrm{m}^{2} \\
2015\end{array}$ & PCOC & $\begin{array}{c}\text { Productive } \\
\text { tillers } / \mathrm{m}^{2} \\
2016\end{array}$ & PCOC & $\begin{array}{c}\text { Grain } \\
\text { wt/panicle } \\
\text { (g) } 2015\end{array}$ & PCOC & $\begin{array}{c}\text { Grain } \\
\text { wt/panicle } \\
\text { (g) } 2016\end{array}$ & PCOC \\
\hline$T_{1}:$ GM + FYM (AWD) & 219 & 0.69 & 228 & 1.56 & 1.54 & 0.40 & 1.54 & -0.22 \\
\hline $\mathrm{T}_{2}$ : Organic (AWD) & 212 & -2.46 & 216 & -3.79 & 1.43 & -6.86 & 1.42 & -7.67 \\
\hline $\mathrm{T}_{3}: \mathrm{FYM}+\mathrm{VC}$ (AWD) & 219 & 0.92 & 222 & -0.97 & 1.56 & 2.03 & 1.55 & 0.34 \\
\hline $\mathrm{T}_{4}$ : SRI with FYM (AWD) & 190 & -12.29 & 192 & -14.14 & 1.61 & 5.04 & 1.67 & 8.37 \\
\hline $\mathrm{T}_{5}:$ DSR + Soybean (LSI) & 230 & 5.76 & 231 & 3.13 & 1.50 & -1.70 & 1.52 & -1.12 \\
\hline $\mathrm{T}_{6}:$ Organic Control (CF) & 214 & -1.38 & 214 & -4.54 & 1.44 & -5.58 & 1.48 & -4.20 \\
\hline $\begin{array}{l}\mathrm{T}_{7} \text { : Chemical Control } \\
\text { (CF) }\end{array}$ & 217 & - & 224 & - & 1.53 & - & 1.54 & - \\
\hline $\mathrm{T}_{8}$ : Integrated (CF) & 220 & 1.38 & 231 & 3.13 & 1.58 & 3.49 & 1.57 & 1.62 \\
\hline $\mathrm{CD}(\mathrm{p}=0.05)$ & \multicolumn{2}{|l|}{8.58} & \multicolumn{2}{|c|}{11.95} & \multicolumn{2}{|l|}{ NS } & \multicolumn{2}{|l|}{0.11} \\
\hline SEm \pm & \multicolumn{2}{|l|}{2.83} & \multicolumn{2}{|l|}{3.94} & \multicolumn{2}{|l|}{0.03} & \multicolumn{2}{|c|}{0.04} \\
\hline \multicolumn{9}{|l|}{ Variety } \\
\hline Type-3 Basmati & \multicolumn{2}{|l|}{218} & \multicolumn{2}{|l|}{222} & \multicolumn{2}{|c|}{1.51} & \multicolumn{2}{|c|}{1.52} \\
\hline Taraori Basmati & \multicolumn{2}{|l|}{212} & \multicolumn{2}{|l|}{217} & \multicolumn{2}{|c|}{1.53} & \multicolumn{2}{|c|}{1.55} \\
\hline $\mathrm{CD}(\mathrm{p}=0.05)$ & \multirow{2}{*}{\multicolumn{2}{|c|}{$\begin{array}{l}3.12 \\
1.04\end{array}$}} & \multirow{2}{*}{\multicolumn{2}{|c|}{$\begin{array}{l}4.15 \\
1.38\end{array}$}} & \multicolumn{2}{|l|}{ NS } & \multicolumn{2}{|l|}{ NS } \\
\hline $\mathrm{SEm} \pm$ & & & & & \multicolumn{2}{|c|}{0.01} & \multicolumn{2}{|c|}{0.01} \\
\hline
\end{tabular}

PCOC: Percent change over chemical control

Table.4 Effect of different organic, inorganic and integrated nutrient sources on thousand grain weight (g) and Number of filled grains/panicle in 2015 and 2016

\begin{tabular}{|c|c|c|c|c|c|c|c|c|}
\hline Treatments & $\begin{array}{c}1000 \text { grain } \\
\text { weight }(\mathrm{g}) \\
2015\end{array}$ & PCOC & $\begin{array}{c}1000 \\
\text { grain } \\
\text { weight } \\
\text { (g) } 2016\end{array}$ & PCOC & $\begin{array}{c}\text { No of filled } \\
\text { grains/panicle } \\
2015\end{array}$ & PCOC & $\begin{array}{c}\text { No of filled } \\
\text { grains/panicle } \\
2016\end{array}$ & PCOC \\
\hline $\begin{array}{l}T_{1}: G M+F Y M \\
(A W D)\end{array}$ & 20.98 & -0.06 & 21.10 & 0.19 & 121 & 22.29 & 109 & 9.72 \\
\hline $\mathrm{T}_{2}$ : Organic (AWD) & 21.06 & 0.33 & 20.96 & -0.47 & 108 & 8.95 & 99 & -0.17 \\
\hline$T_{3}: F Y M+V C(A W D)$ & 21.37 & 1.81 & 20.97 & -0.41 & 114 & 15.71 & 111 & 11.89 \\
\hline $\begin{array}{l}T_{4}: \text { SRI with FYM } \\
\text { (AWD) }\end{array}$ & 20.83 & -0.75 & 21.08 & 0.09 & 127 & 28.37 & 123 & 23.45 \\
\hline $\begin{array}{l}\text { T}_{5}: \text { DSR + Soybean } \\
\text { (LSI) }\end{array}$ & 20.96 & -0.17 & 20.87 & -0.91 & 117 & 18.75 & 92 & -7.20 \\
\hline $\begin{array}{l}\mathrm{T}_{6} \text { : Organic Control } \\
(\mathrm{CF})\end{array}$ & 20.97 & -0.08 & 21.05 & -0.04 & 103 & 3.88 & 106 & 6.20 \\
\hline $\begin{array}{l}\mathrm{T}_{7} \text { : Chemical Control } \\
\text { (CF) }\end{array}$ & 20.99 & - & 21.06 & - & 99 & - & 100 & - \\
\hline$T_{8}:$ Integrated $(\mathrm{CF})$ & 21.37 & 1.81 & 21.07 & 0.06 & 118 & 19.73 & 114 & 14.57 \\
\hline $\mathrm{CD}(\mathrm{p}=0.05)$ & \multicolumn{2}{|c|}{0.44} & \multicolumn{2}{|c|}{ NS } & \multicolumn{2}{|l|}{ NS } & \multicolumn{2}{|l|}{ NS } \\
\hline $\mathrm{SEm} \pm$ & \multicolumn{2}{|c|}{0.15} & \multicolumn{2}{|c|}{0.16} & \multicolumn{2}{|l|}{13.57} & \multicolumn{2}{|l|}{6.64} \\
\hline \multicolumn{9}{|l|}{ Variety } \\
\hline Type-3 Basmati & \multicolumn{2}{|c|}{20.41} & \multicolumn{2}{|c|}{20.59} & \multicolumn{2}{|l|}{128} & \multicolumn{2}{|l|}{120} \\
\hline Taraori Basmati & \multicolumn{2}{|c|}{21.72} & \multicolumn{2}{|c|}{21.46} & \multicolumn{2}{|l|}{98} & \multicolumn{2}{|l|}{94} \\
\hline $\mathrm{CD}(\mathrm{p}=0.05)$ & \multicolumn{2}{|c|}{0.34} & \multicolumn{2}{|c|}{0.172} & \multicolumn{2}{|l|}{17} & \multicolumn{2}{|l|}{10.35} \\
\hline $\mathrm{SEm} \pm$ & \multicolumn{2}{|c|}{0.11} & \multicolumn{2}{|c|}{0.58} & \multicolumn{2}{|l|}{5.55} & \multicolumn{2}{|l|}{3.45} \\
\hline
\end{tabular}

PCOC: Percent change over chemical control 
Table.5 Effect of different organic, inorganic and integrated nutrient sources on grain yield (t/ha), straw yield (t/ha) and harvest index (\%) of two basmati rice varieties in 2015

\begin{tabular}{|c|c|c|c|c|c|c|}
\hline Treatments & $\begin{array}{c}\text { Grain yield } \\
\text { (t/ha) }\end{array}$ & PCOC & $\begin{array}{l}\text { Straw } \\
\text { yield } \\
\text { (t/ha) }\end{array}$ & PCOC & $\begin{array}{c}\text { Harvest index } \\
(\%)\end{array}$ & PCOC \\
\hline $\mathrm{T}_{1}: \mathrm{GM}+\mathrm{FYM}(\mathrm{AWD})$ & 2.97 & 4.85 & 5.35 & 3.65 & 35.68 & 0.74 \\
\hline $\mathrm{T}_{2}:$ Organic (AWD) & 2.64 & -6.73 & 4.46 & -13.57 & 37.19 & 4.99 \\
\hline$T_{3}: F Y M+V C(A W D)$ & 2.98 & 5.24 & 5.47 & 5.95 & 35.29 & -0.35 \\
\hline T $_{4}$ : SRI with FYM (AWD) & 2.66 & -6.02 & 4.58 & -11.24 & 36.76 & 3.77 \\
\hline $\mathrm{T}_{5}:$ DSR + Soybean (LSI) & 2.94 & 3.90 & 4.95 & -4.15 & 37.28 & 5.25 \\
\hline $\mathrm{T}_{6}$ : Organic Control (CF) & 2.73 & -3.53 & 4.93 & -4.37 & 35.63 & 0.58 \\
\hline $\mathrm{T}_{7}$ : Chemical Control (CF) & 2.83 & - & 5.16 & - & 35.42 & - \\
\hline $\mathrm{T}_{8}$ : Integrated (CF) & 2.98 & 5.41 & 5.50 & 6.53 & 35.19 & -0.64 \\
\hline $\mathrm{CD}(\mathrm{p}=0.05)$ & \multicolumn{2}{|l|}{0.21} & \multicolumn{2}{|c|}{0.33} & \multicolumn{2}{|l|}{0.67} \\
\hline SEm \pm & \multicolumn{2}{|c|}{0.06} & \multicolumn{2}{|c|}{0.11} & \multicolumn{2}{|l|}{0.22} \\
\hline \multicolumn{7}{|l|}{ Variety } \\
\hline Type-3 Basmati & \multicolumn{2}{|c|}{2.86} & \multicolumn{2}{|c|}{5.16} & \multicolumn{2}{|l|}{35.66} \\
\hline Taraori Basmati & \multicolumn{2}{|c|}{2.83} & \multicolumn{2}{|c|}{4.94} & \multicolumn{2}{|l|}{36.44} \\
\hline $\mathrm{CD}(\mathrm{p}=0.05)$ & \multicolumn{2}{|c|}{ NS } & \multicolumn{2}{|c|}{0.14} & \multicolumn{2}{|l|}{0.71} \\
\hline SEm \pm & \multicolumn{2}{|c|}{0.02} & \multicolumn{2}{|c|}{0.05} & \multicolumn{2}{|l|}{0.24} \\
\hline
\end{tabular}

PCOC: Percent change over chemical control

Table.6 Effect of different organic, inorganic and integrated nutrient sources on grain yield (t/ha), straw yield (t/ha) and harvest index (\%) of two basmati rice varieties in 2016.

\begin{tabular}{|c|c|c|c|c|c|c|}
\hline Treatments & $\begin{array}{l}\text { Grain yield } \\
\text { (t/ha) }\end{array}$ & PCOC & $\begin{array}{c}\text { Straw } \\
\text { yield } \\
\text { (t/ha) }\end{array}$ & PCOC & $\begin{array}{c}\text { Harvest } \\
\text { index }(\%)\end{array}$ & PCOC \\
\hline $\mathrm{T}_{1}: \mathrm{GM}+\mathrm{FYM}(\mathrm{A}$ WD) & 2.99 & 3.24 & 5.68 & 3.49 & 34.52 & -0.24 \\
\hline $\mathrm{T}_{2}:$ Organic (A WD) & 2.52 & -13.24 & 4.86 & -11.48 & 34.11 & -1.40 \\
\hline$T_{3}: F Y M+V C(A W D)$ & 2.88 & -0.58 & 5.55 & 1.10 & 34.20 & -1.14 \\
\hline $\begin{array}{l}\text { T}_{4}: \text { SRI with FYM } \\
\text { (AWD) }\end{array}$ & 2.64 & -9.09 & 4.91 & -10.51 & 34.99 & 1.13 \\
\hline$T_{5}:$ DSR + Soybean (LSI) & 3.27 & 12.85 & 5.28 & -3.85 & 38.25 & 10.54 \\
\hline $\mathrm{T}_{6}$ : Organic Control (CF) & 2.61 & -9.93 & 5.27 & -4.06 & 33.16 & -4.17 \\
\hline $\begin{array}{l}\mathrm{T}_{7} \text { : Chemical Control } \\
(\mathrm{CF})\end{array}$ & 2.90 & - & 5.49 & - & 34.60 & - \\
\hline $\mathrm{T}_{8}$ : Integrated (CF) & 3.00 & 3.52 & 5.71 & 4.01 & 34.46 & -0.40 \\
\hline $\mathrm{CD}(\mathrm{p}=0.05)$ & \multicolumn{2}{|l|}{0.18} & \multicolumn{2}{|c|}{0.29} & \multicolumn{2}{|c|}{1.31} \\
\hline $\operatorname{SEm} \pm$ & \multicolumn{2}{|l|}{0.06} & \multicolumn{2}{|c|}{0.10} & \multicolumn{2}{|c|}{0.43} \\
\hline \multicolumn{7}{|l|}{ Variety } \\
\hline Type-3 Basmati & \multicolumn{2}{|l|}{2.89} & \multicolumn{2}{|c|}{5.42} & \multicolumn{2}{|c|}{34.76} \\
\hline Taraori Basmati & \multicolumn{2}{|l|}{2.81} & \multicolumn{2}{|c|}{5.26} & \multicolumn{2}{|c|}{34.81} \\
\hline $\mathrm{CD}(\mathrm{p}=0.05)$ & \multicolumn{2}{|l|}{0.06} & \multicolumn{2}{|c|}{0.15} & \multicolumn{2}{|c|}{ NS } \\
\hline SEm \pm & \multicolumn{2}{|l|}{0.02} & \multicolumn{2}{|c|}{0.05} & \multicolumn{2}{|c|}{0.24} \\
\hline
\end{tabular}

PCOC: Percent change over chemical control 
Similarly application of $50 \% \mathrm{~N}$ through RDF $+50 \% \mathrm{~N}$ through vermicompost recorded higher 1000-grain weight (19.7 g) (Dekhane et al., 2014). In another study highest test weight $(22.32 \mathrm{~g})$ was recorded in SRI treatment followed by DSR (21.84 g) which was significantly higher with organic control $(19.88 \mathrm{~g})$ and chemical control $(20.00 \mathrm{~g})$ treatment during 2012. While, in 2013, highest test weight was recorded in GM + VC treatment $(23.33 \mathrm{~g})$ which was significantly higher with DSR (19.70 g) and organic control (19.73 g) treatment. However, there was no significant difference in all treatments during 2014 (Singh et al., 2017).

\section{Number of filled grains/panicle}

Number of filled grains/panicle of both the basmati rice varieties under different organic, inorganic and integrated treatments in 2015 and 2016 are summarized in Table 4. In 2015 maximum number of filled grains/panicle (127) were observed in $\mathrm{T}_{4}$ SRI with FYM (AWD) and minimum (99) in $\mathrm{T}_{7}$ Chemical Control (CF). The number of filled grains/panicle in the treatments followed the order $\mathrm{T}_{4} \mathrm{SRI}$ with FYM (AWD) $>\mathrm{T}_{1} \mathrm{GM}+$ FYM (AWD) $>T_{8}$ Integrated (CF) $>T_{5}$ DSR + Soybean $(\mathrm{LSI})>\mathrm{T}_{3} \mathrm{FYM}+\mathrm{VC}(\mathrm{AWD})>\mathrm{T}_{2}$ Organic (AWD) $>\mathrm{T}_{6}$ Organic Control $(\mathrm{CF})>$ $\mathrm{T}_{7}$ Chemical Control (CF). The maximum percent increase in number of filled grains/panicle $(28.37 \%)$ was observed in $\mathrm{T} 4$ SRI with FYM (AWD) over $\mathrm{T}_{7}$ Chemical Control (CF) and minimum percent increase $(3.88 \%)$ in number of filled grains/panicle was found in $\mathrm{T}_{6}$ Organic Control (CF) over $\mathrm{T}_{7}$ Chemical Control (CF). However among both the basmati rice varieties higher number of filled grains/panicle (128) was found in Type3 than Taraori (98) basmati rice variety.

In 2016 maximum number of filled grains/panicle (123) was observed in $\mathrm{T}_{4} \mathrm{SRI}$ with FYM (AWD) and minimum (92) in $\mathrm{T}_{5}$
DSR + Soybean (LSI). The number of filled grains/panicle in the treatments followed the order $\mathrm{T}_{4}$ SRI with FYM (AWD) $>\mathrm{T}_{8}$ Integrated $(\mathrm{CF})>\mathrm{T}_{3} \mathrm{FYM}+\mathrm{VC}(\mathrm{AWD})>\mathrm{T}_{1}$ $\mathrm{GM}+\mathrm{FYM}(\mathrm{AWD})>\mathrm{T}_{6}$ Organic Control (CF) $>\mathrm{T}_{7}$ Chemical Control (CF) $>\mathrm{T}_{2}$ Organic (AWD) $>\mathrm{T}_{5}$ DSR + Soybean (LSI). The maximum percent increase in number of filled grains/panicle $(23.45 \%)$ was observed in $\mathrm{T}_{4}$ SRI with FYM (AWD) over $\mathrm{T}_{7}$ Chemical Control (CF) and maximum percent decrease $(-7.20 \%)$ in number of filled grains/panicle was found in $\mathrm{T}_{5}$ DSR + Soybean (LSI) over $\mathrm{T}_{7}$ Chemical Control (CF). However among both the basmati rice varieties higher number of filled grains/panicle (120) was found in Type-3 than Taraori (94) basmati rice variety. The number of grains per panicle contributes materially towards the final grain yield. It was reported that maximum filled grains per panicle (138.64) was observed in $\mathrm{T}_{2}$ (100\% RDF) which was statistically at par with poultry manure $+50 \%$ of RDF (138.06) and compost $+50 \%$ of RDF (137.55) (Arif et al., 2014). It may be due to the reason that SRI has wider spacing between the plants due to which there is less below and above ground competitions for better grain filling and more number of filled grains per panicle. Subsequently resulting in better source to sink conversion and in turn enhanced the production of more total number of seeds and filled seeds per panicle (Lu et al., 2005).

In a similar experiment application of $50 \% \mathrm{~N}$ through RDF $+50 \% \mathrm{~N}$ through vermicompost also recorded higher number of grains per panicle (128.0) (Dekhane et al., 2014). The significantly higher number of filled grains per panicle (220.2) was recorded in SRI method as compared to conventional method (138.0) in Dehradun rice variety (Mandal et al., 2015). The $\mathrm{O}_{\mathrm{p}}$ treatment produced the highest filled grain percentage in both the dry $(88.18 \%)$ and wet $(80.67 \%)$ seasons but these 
values did not differ significantly from those recorded for the $\mathrm{O}_{c}$ and $\mathrm{O}_{\mathrm{v}}$ treatments. The $\mathrm{O}_{0}$ treatment produced the lowest percentages in both season (Moe et al., 2017).

\section{Grain yield, straw yield and harvest index}

Grain yield, straw yield and harvest index of both the basmati rice varieties under different organic, inorganic and integrated treatments in both the years are summarized in Figure 2 and Table $5 \& 6$.

\section{Grain yield (t/ha)}

The grain yield in 2015 was maximum (2.98 $\mathrm{t} / \mathrm{ha}$ ) in $\mathrm{T}_{8}$ Integrated $(\mathrm{CF})$ and $\mathrm{T}_{3} \mathrm{FYM}+\mathrm{VC}$ (AWD) $(2.98 \mathrm{t} / \mathrm{ha})$ and minimum $(2.64 \mathrm{t} / \mathrm{ha})$ in $\mathrm{T}_{2}$ Organic (AWD). The grain yield in the treatments followed the order $\mathrm{T}_{8}$ Integrated $(\mathrm{CF})=\mathrm{T}_{3} \mathrm{FYM}+\mathrm{VC}(\mathrm{AWD})>\mathrm{T}_{1} \mathrm{GM}+$ FYM $($ AWD $)>\mathrm{T}_{5}$ DSR + Soybean (LSI) > $\mathrm{T}_{7}$ Chemical Control (CF) $>\mathrm{T}_{6}$ Organic Control $(\mathrm{CF})>\mathrm{T}_{4}$ SRI with FYM $($ AWD) $>$ $\mathrm{T}_{2}$ Organic (AWD). The maximum percent increase in grain yield $(5.41 \%)$ was observed in $\mathrm{T}_{8}$ Integrated $(\mathrm{CF})$ over $\mathrm{T}_{7}$ Chemical Control (CF) and maximum percent decrease $(-6.73 \%)$ in grain yield was found in $\mathrm{T}_{2}$ Organic (AWD) over $\mathrm{T}_{7}$ Chemical Control $(\mathrm{CF})$. However among both the basmati rice varieties higher grain yield (2.86 t/ha) was found in Type-3 than Taraori (2.83 t/ha) basmati rice variety.

In the year 2016 maximum grain yield $(3.00$ t/ha) was observed in $\mathrm{T}_{8}$ Integrated $(\mathrm{CF})$ and minimum (2.52 t/ha) in $\mathrm{T}_{2}$ Organic (AWD). The grain yield in the treatments followed the order $\mathrm{T}_{5} \mathrm{DSR}+$ Soybean (LSI) $>\mathrm{T}_{8}$ Integrated $(\mathrm{CF})>\mathrm{T}_{1} \mathrm{GM}+\mathrm{FYM}(\mathrm{AWD})>$ $\mathrm{T}_{7}$ Chemical Control $(\mathrm{CF})>\mathrm{T}_{3} \mathrm{FYM}+\mathrm{VC}$ $\left(\right.$ AWD) $>\mathrm{T}_{4}$ SRI with FYM $\left(\right.$ AWD) $>\mathrm{T}_{6}$ Organic Control (CF) $>\mathrm{T}_{2}$ Organic (AWD).The highest percent increase in grain yield $(12.85 \%)$ was observed in $\mathrm{T}_{5} \mathrm{DSR}+$ Soybean (LSI) over $\mathrm{T}_{7}$ Chemical Control
(CF) and maximum percent decrease ($13.24 \%$ ) in grain yield was found in $T_{2}$ Organic (AWD) over $\mathrm{T}_{7}$ Chemical Control (CF). However among both the basmati rice varieties higher grain yield $(2.89 \mathrm{t} / \mathrm{ha})$ was found in Type-3 than Taraori (2.81 t/ha) basmati rice variety.

\section{Straw yield (t/ha)}

The straw yield in 2015 was maximum (5.50 $\mathrm{t} / \mathrm{ha})$ in $\mathrm{T}_{8}$ Integrated $(\mathrm{CF})$ and minimum (4.46 t/ha) in $T_{2}$ Organic (AWD). The straw yield in the treatments followed the order $\mathrm{T}_{5}$ DSR + Soybean (LSI) $>\mathrm{T}_{4}$ SRI with FYM $\left(\right.$ AWD) $>\mathrm{T}_{7}$ Chemical Control (CF) $>\mathrm{T}_{1}$ GM+FYM (AWD) $>\mathrm{T}_{8}$ Integrated $(\mathrm{CF})>\mathrm{T}_{3}$ $\mathrm{FYM}+\mathrm{VC}(\mathrm{AWD})>\mathrm{T}_{2}$ Organic $(\mathrm{AWD})>\mathrm{T}_{6}$ Organic Control (CF). The maximum percent increase in straw yield $(6.53 \%)$ was observed in $\mathrm{T}_{8}$ Integrated $(\mathrm{CF})$ over $\mathrm{T}_{7}$ Chemical Control (CF) and maximum percent decrease $(-13.57 \%)$ in straw yield was found in $\mathrm{T}_{2}$ Organic (AWD) over $\mathrm{T}_{7}$ Chemical Control $(\mathrm{CF})$. However among both the basmati rice varieties higher straw yield (5.16 t/ha) was found in Type-3 than Taraori (4.94 t/ha) basmati rice variety.

In the year 2016 maximum straw yield (5.71 $\mathrm{t} / \mathrm{ha}$ ) was observed in $\mathrm{T}_{8}$ Integrated $(\mathrm{CF})$ and minimum (4.86 t/ha) in $\mathrm{T}_{2}$ Organic (AWD).The straw yield in the treatments followed the order $\mathrm{T}_{8}$ Integrated $(\mathrm{CF})>\mathrm{T}_{1}$ $\mathrm{GM}+\mathrm{FYM}(\mathrm{AWD})>\mathrm{T}_{3} \mathrm{FYM}+\mathrm{VC}(\mathrm{AWD})$ $>\mathrm{T}_{7}$ Chemical Control $(\mathrm{CF})>\mathrm{T}_{5} \mathrm{DSR}+$ Soybean (LSI) $>\mathrm{T}_{6}$ Organic Control $(\mathrm{CF})>$ $\mathrm{T}_{4}$ SRI with FYM (AWD) $>\mathrm{T}_{2}$ Organic (AWD). The highest percent increase in straw yield $(4.01 \%)$ was observed in $\mathrm{T}_{8}$ Integrated (CF) over $\mathrm{T}_{7}$ Chemical Control $(\mathrm{CF})$ and maximum percent decrease $(-11.48 \%)$ in straw yield was found in $\mathrm{T}_{2}$ Organic (AWD) over $\mathrm{T}_{7}$ Chemical Control (CF). However among both the basmati rice varieties higher straw yield (5.42 t/ha) was found in Type-3 than Taraori (5.26 t/ha) basmati rice variety. 
The higher yields in the integrated treatment could be due to the reason that there might be continuous supply of nutrients throughout the crop growth period (Aulakh et al., 2016). In a similar experiment rice grown through conventional tansplanting (CT) gave significantly higher grain and straw yields as compared to that grown through SRI. Grain yield of rice was significantly influenced by INM as well as application of inorganic fertilizer over the control. The grain yields ( 4.44 to $4.23 \mathrm{t} / \mathrm{ha}$ ) were recorded with the recommended doses of chemical fertilizers which were statistically at par in the INM treatments. However, grain yield and straw yield were higher in INM as compared to the recommended doses of chemical fertilizers (Singh et al., 2013).

In another experiment also the application of $50 \% \mathrm{~N}$ through RDF $+50 \% \mathrm{~N}$ through vermicompost recorded higher grain yield (4.97 t/ha) and straw yield (5.77 t/ha) of rice variety GR 11 . Minimum grain yield (2.76 $\mathrm{t} / \mathrm{ha}$ ) and straw yield (3.53 t/ha) was in control with rice variety GR 11 (Dekhane et al., 2014). Significantly higher grain yield (54 $\mathrm{q} / \mathrm{ha})$, straw yield $(70 \mathrm{q} / \mathrm{ha})$ were recorded under 100\% RDF + vermicompost @ $5 \mathrm{mt} / \mathrm{ha}$ +Trichoderma compost @ 7.5 kg/ha (Shukla et al., 2016).

Rice produced higher grain yield with different $\mathrm{N}$ source ratios. Among the three ratios of urea and organic sources, 50\% urea and 50\% organic sources resulted in the highest grain yield $\left(9417 \mathrm{~kg} / \mathrm{hm}^{2}\right)$, followed by $75 \%$ urea and $25 \%$ organic sources (9096 $\mathrm{kg} / \mathrm{hm}^{2}$ ), and $25 \%$ urea and $75 \%$ organic sources $\left(9014 \mathrm{~kg} / \mathrm{hm}^{2}\right)$. On average, application of $\mathrm{N}$ mixtures produced higher grain yield $\left(9176 \mathrm{~kg} / \mathrm{hm}^{2}\right)$ than applying pure organic source. There was no significant difference in grain yield with the application of animal manure and crop residue (Amanullah, 2016).
In an another study where integrated use of manure with fertilizers gave on an average 8.3-33.8\% and 2.9-18.3\% higher grain yield in Boro and T. Aman rice, respectively over sole fertilizers treatments (Bilkis et al., 2017).

In a similar study all the treatments exerted significant influence on the yield of rice. Among the treatments, application of vermicompost @ 5t ha ${ }^{-1}$ with Azospirillum and phosphobacteria was superior and recorded the highest yield of rice. Treatment $\mathrm{S}_{3}$ (Press mud @ 10t ha ${ }^{-1}$ with Azospirillum and phospho bacteria) was next in order of ranking. The least yield of rice was recorded in the treatment with no organic manure and biofertilizers $\left(\mathrm{S}_{5}\right)$ (Raman and Prakash, 2017).

\section{Harvest index (\%)}

The harvest index in 2015 was maximum $(37.28 \%)$ in $\mathrm{T}_{5}$ DSR + Soybean (LSI) and minimum (35.29\%) in $\mathrm{T}_{3} \mathrm{FYM}+\mathrm{VC}$ (AWD). The harvest index in the treatments followed the order $\mathrm{T}_{5} \mathrm{DSR}+$ Soybean (LSI) $>\mathrm{T}_{2}$ Organic (AWD) $>\mathrm{T}_{4}$ SRI with FYM (AWD) $\mathrm{T}_{1} \mathrm{GM}+\mathrm{FYM}(\mathrm{AWD})>\mathrm{T}_{6}$ Organic Control (CF) $>\mathrm{T}_{7}$ Chemical Control (CF) $>\mathrm{T}_{3}$ FYM+VC (AWD) $>T_{8}$ Integrated (CF). The maximum percent increase in harvest index $(5.25 \%)$ was observed in $\mathrm{T}_{5}$ DSR + Soybean (LSI) over $\mathrm{T}_{7}$ Chemical Control (CF) and maximum percent decrease $(-0.64 \%)$ in harvest index was found in $\mathrm{T}_{8}$ Integrated $(\mathrm{CF})$ over $\mathrm{T}_{7}$ Chemical Control (CF). However among both the basmati rice varieties higher harvest index was found in Taraori $(36.44 \%)$ than Type-3 (35.66 \%) basmati rice variety.

In the year 2016 maximum harvest index $(38.25 \%)$ was observed in $\mathrm{T}_{5} \mathrm{DSR}+$ Soybean (LSI) and minimum (33.16 \%) in $\mathrm{T}_{6}$ Organic Control (CF). The harvest index in the treatments followed the order $\mathrm{T}_{5}$ DSR + Soybean $(\mathrm{LSI})>\mathrm{T}_{4}$ SRI with FYM $(\mathrm{AWD})>$ $\mathrm{T}_{7}$ Chemical Control (CF) $>\mathrm{T}_{1} \mathrm{GM}+\mathrm{FYM}$ 
$\left(\right.$ AWD) $>\mathrm{T}_{8}$ Integrated $(\mathrm{CF})>\mathrm{T}_{3} \mathrm{FYM}+\mathrm{VC}$ $\left(\right.$ AWD) $>T_{2}$ Organic (AWD) $>T_{6}$ Organic Control (CF). The highest percent increase in harvest index $(10.54 \%)$ was observed in $\mathrm{T}_{5}$ DSR + Soybean (LSI) over $\mathrm{T}_{7}$ Chemical Control (CF) and maximum percent decrease $(-4.17 \%)$ in harvest index was found in $\mathrm{T}_{6}$ Organic Control (CF) over $\mathrm{T}_{7}$ Chemical Control (CF). However among both the basmati rice varieties higher harvest index (34.81\%) was found in Taraori than Type-3 $(34.76 \%)$ basmati rice variety.

In a similar experiment harvest index of Pusa 44 was significantly higher as compared to that of PB1, but it was not significantly influenced due to difference in crop nutrition (Singh et al., 2013). Harvest index shows that there were significant differences among different manures. Poultry manure @ 10t/ha + $50 \%$ of RDF had highest HI (43.76\%) while control had lowest harvest index. Higher yield and harvest index of poultry manure $+50 \%$ of RDF indicates better partitioning of photosynthetic substance to economic yield (Arif et al., 2014).

The harvest index (HI) ranged from 0.42 to 0.56 for both inorganic fertilizer and organic manure treatments and there were significant differences among them. However, similar HI values were measured among $\mathrm{I}_{50}, \mathrm{I}_{75}$, and $\mathrm{I}_{100}$ treatments throughout both seasons. For the organic manures, the $\mathrm{O}_{\mathrm{p}}$ treatment produced the highest HI values. These were 0.54 and 0.51 in the dry and wet seasons, respectively. The lowest HI values were recorded for the $\mathrm{I}_{0} \mathrm{O}_{0}$ treatment (Moe et al., 2017).

In conclusion the application of organic manures such as FYM and VC as well as green manure Sesbania aculeata were effective in enhancing growth, yield, and the yield components of the two basmati rice varieties (Type-3 and Taraori).
These results have immense potential for reducing the use of chemical fertilizers without decreasing the yield of the basmati rice varieties.

Since the introduction of high yielding varieties and increased use of chemical fertilizers and pesticides have lead to several harmful effects on the soil environment ultimately reducing the productivity of the soil by affecting the soil health.

It is felt that organic farming may solve all these problems by protecting/sustaining soil health. A major advantage of using organic wastes is that they not only act as the source of nutrients, but also provide micronutrients and enhance the microbial population of soils which help in mobilizing native nutrients in soil-plant system and also improve the efficiency of applied nutrients at very low cost.

Further studies on how various other different organic fertilizers such as poultry manure, fish manure etc and their combination might enhance the growth and yield of recently introduced basmati rice varieties might be performed in the future.

\section{References}

Adjao, R.T. and Staatz, J.M. 2015. Asian rice economy changes and implications for sub-Saharan Africa. Global Food Security. 5: 50-55.

Amanullah, H. 2016. Influence of organic and inorganic nitrogen on grain yield and yield components of hybrid rice in Northwestern Pakistan. Rice Science. 23: 326-333.

Arif, M., Tasneem, M., Bashir, F., Yaseen, G. and Iqbal, R.M. 2014. Effect of integrated use of organic manures and inorganic fertilizers on yield and yield components of rice. Journal of agricultural research. 52(2): 197-206. 
Bana, O.P.S. and Pant, A.K. 2000. Green manuring for ecologically sound crop production. Indian Farmers' Digest, 33:19-20.

Bilkis, S., Islam, M.R., Jahiruddin, M. and Rahaman, M.M. 2017. Integrated use of manure and fertilizers increases rice yield, nutrient uptake and soil fertility in the boro-fallow-t. aman rice cropping pattern. SAARC Journal of Agriculture. 15(2):147-161. Das, D.K. and Mandal, M. Advanced Technology of Fertilizer Uses for Crop Production. pp. 18-68.

Dekhane, S.S., Patel, D.J., Jadhav, P.B., Kireeti, A., Patil, N.B., Harad, N.B., Jadhav, K.P. 2014. Effect of organic and inorganic fertilizer on growth and yield of paddy cv GR 11. International Journal of Information Research and Review. 1: 2628.

Gopalakrishnan, S., Srinivas, V., Alekhya, G., Prakash, B., Kudapa, H., Rathore, A. and Varshney, R.K. 2015. The extent of grain yield and plant growth enhancement by plant growth-promoting broad-spectrum Streptomyces sp. in chickpea. SpringerPlus. 4(1): 31.

Gross, B.L., Zhao, Z. 2014. Archaeological and genetic insights into the origins of domesticated rice. Proceedings of the National Academy of Sciences. 111: 6190-6197.

IRRI, 2006. Bringing hope, improving livesstrategies. Plan 2007-2015. International Rice Research Institute, Los Banos, pp. 61.

Khakwani, A.A., Saddozai, U.K., Awan, I.U., Baloch, M.S., Khan, Q., Munir, M., Saddozai, G., Bakhsh, I. 2016. Effect of organic and inorganic inputs on soil parameters and productivity of coarse rice (oryza sativa 1. CV. IR-9). Journal of agricultural research. 54: 195-205.

Khidrapure, G., Uppar, D.S., Maruti, K., Tejagouda, M.B., Shankrayya. 2015. Effect of integrated nutrient management on plant growth and seed yield in hybrid maize. The Bioscan.10: 369-371.
Kumar, A.R., Balusamy, M. 2017. Integrated use of organic and inorganic sources of nutrients on growth and yield of rice (Oryza sativa L.). Journal of Pharmacognosy and Phytochemistry. 6: 1416-1419.

Lori, M., Symnaczik, S., Mäder, P., De Deyn, G., Gattinger, A. 2017. Organic farming enhances soil microbial abundance and activity - A meta-analysis and metaregression. PLoS One. 12: 0180442.

Lu, Y., Li, J., Wang, J., Tang, Y. and Yu, G. 2005. Effects of SRI on dry matter prodution and grain yield of Yuyou 11. Southwest China journal of agricultural sciences. 18(6): 719-723.

Mandal, K., Pramanick, M. and Bandopadhayay, P. 2014. Influence of crop establishment systems on yield and quality of rice grain and seeds of aromatic rice (Oryza sativa L.). International Journal of Applied Agricultural \& Horticultural Sciences. 951-955.

Moe, K., Mg, K.W., Win, K.K. Yamakawa, T. 2017. Combined effect of organic manures and inorganic fertilizers on the growth and yield of hybrid rice (Palethwe-1). American Journal of Plant Sciences. 8: 1022.

Norman, J.M., Campbell, G.S. 1989. Canopy structure. In Plant physiological ecology (301-325). Springer Dordrecht.

Oo, A.N., Banterng, P., Polthanee, A., TreloGes, V. 2010. The effect of different fertilizers management strategies on growth and yield of upland black glutinous rice and soil property. Asian Journal of Plant Sciences. 9: 414.

Raman, R. and Prakash, P. 2017. Sustainable rice production system as influenced by organic manures and biofertilizers. Journal of Agricultural Research. 2(2): 000126.

Reganold, J.P. and Wachter, J.M. 2016. Organic agriculture in the twenty-first century. Nature Plants. 2: 15221.

Ricepedia. Asia. [Online]Available from: http://ricepedia.org/rice-around-the- 
world/asia. [Assessed on 18 November 2017].

Shukla, S., Meena, R.N., Meena, R., Verma, V.K., Ghilotia, Y.K. and Gaurav. 2016. Effects of different organic sources of nutrition on nutrient uptake, yield attributes and economics of Oryza sativa L. 445-449.

Singh, A.B. and Ganguly, T.K., 2005. Quality comparison of conventional compost, vermicompost and chemically-enriched compost. Journal of the Indian Society of Soil Science. 53(3):352-355.

Singh, D.K., Akhtar, Z., Gupta, S., Srivastava, A., Chakraborty, M. 2017. Production strategies of organic basmati rice in Tarai region of Uttarakhand, India. Organic Agriculture. 7: 21-30.

Singh, Y.V., Singh, K.K. and Sharma, S.K., 2013. Influence of crop nutrition on grain yield, seed quality and water productivity under two rice cultivation systems. Rice Science. 20(2):129-138.

Srivastava, V.K., Singh, J.K. and Vishwakarma, A. 2016. Effect of fertility levels and mode of nitrogen nutrition on productivity and profitability of hybrid rice under system of rice intensification. International Journal of Agriculture Sciences. 8(47): 1983-1986.

Thiyagarajan, T.M., Hengsdijk, H. and Bindraban, P.S. 2005. Transitions in agriculture for enhancing water productivity: proceedings of an international symposium held in Killikulam, Tamil Nadu, India, 23-25
September 2003. Agricultural College and Research Institute.

Tiwari, R., Upadhyay, A.K., Rai, H.K., Sachidanand, B., Pradip, D., Rohit, P. 2018. Influence of soil test based integrated fertilization on growth, productivity and quality parameters of rice in Central India. International journal of chemical studies. 6: 10911094.

Usman, M.U.H.A.M.M.A.D., Ullah, E., Warriach, E.A., Farooq, M. and Liaqat, A., 2003. Effect of organic and inorganic manures on growth and yield of rice variety "Basmati 2000". International Journal of Agriculture and Biology. 5(4): 481-483.

Vaithiyanathan, T., Sundaramoorthy, P. 2016. Impact of Organic Manure and Inorganic Fertilizers on Seed Germination of Green Gram (Vigna radiata L.). World Scientific News. 35: 111.

Wheeler, T., Von Braun, J. 2013. Climate change impacts on global food security. Science, 341: 508-513.

Willer, H., Lernoud J. 2017. The world of organic agriculture. Statistics and emerging trends. Research Institute of Organic Agriculture FiBL and IFOAM Organics International. 1-340.

Zarate-Valdez, J.L., Whiting, M.L., Lampinen, B.D., Metcalf, S., Ustin, S.L. Brown, P.H., 2012. Prediction of leaf area index in almonds by vegetation indexes. Computers and electronics in agriculture. 85: 24-32.

\section{How to cite this article:}

Dipti Bisarya, D. K. Singh, M. K. Nautiyal, Deepti Shankhdhar and Shankhdhar S. C. 2018. Effect of Organic, Inorganic and Integrated Nutrient Sources on the Yield and Its Attributes of Two Basmati Rice Varieties viz Type-3 and Taraori Grown in Tarai Regions of Uttarakhand India. Int.J.Curr.Microbiol.App.Sci. 7(10): 3711-3726. doi: $\underline{\text { https://doi.org/10.20546/ijcmas.2018.710.429 }}$ 